\title{
Diagnostic Reference Data for the Monaural Brain-stem Auditory Evoked Response (BAER)
}

\author{
Cyril D Govender M (Audiology) UDW \\ Department of Speech and Hearing Therapy \\ University of Durban-Westville
}

\begin{abstract}
The objective of the investigation was to establish diagnostic reference data for the normal BAER. BAERs were elicited from the target ( $R$ ) ear using clicks presented at $\overline{7} \overline{0} d B n H L$. Relevant latency and amplitude data were obtained from 60 selected normal hearing Indian undergraduate females $(N=30 ; \bar{X}$ age $=20.33$ years) and male $(N=30 ; \bar{X}$ age $=21.33$ years) students aged between 18 and 25 years $(X$ age $=20.73$ years). Diagnostic reference data were established for the absolute latencies of peaks I to VI; relative latencies of peaks I-III; III-Vand I$V ;$ absolute amplitudes of peaks $I$ and $V$ and the relative amplitude ratio of peaks $V: I$. These results are discussed in terms of the literature and implications for clinical application and further research.
\end{abstract}

\section{OPSOMMING}

Die doel van hierdie ondersoek was om diagnostiese verwysingsdata vir normale ouditief-ontlokte breinstamresponse daar te stel. Hierdie response is van dic regteroor ontlok deur middel van klikgeluide wat by $70 d B n G P$ a angebied is. Toepaslike data vir latentheid en amplitude is vir 60 normaalhorende Indiër voorgraadse studente verkry. Vroulike $(N=30 ; \bar{X}$ ouderdom $=20.33$ jaar $)$ en manlike $(N=30 ; \bar{X}$ ouderdom $=$ 21.33jaar) studente, tussen die ouderdom 18 tot 25 jaar $(X$ ouderdom $=20.73$ jaar $)$ is vir die doel van hierdie studie geselekteer. Diagnostiese verwysingsdata is verkry vir die absolute latenthede van pieke I tot VI; relatiewe latenthede van pieke I-III; III-Ven I-V; absolute amplitude van pieke I en $V$ en die relatiewe amplitude-verhouding van pieke V:I. Hierdie resultate is bespreek met verwysing na die literatuur. Die implikasies vir kliniese toepassing en verdere navorsing is ook aangedui.

\section{INTRODUCTION}

During the past decade and a half, there has been a formidable increase in the use of specialised audiological test procedures in otoneurological diagnosis. Since the initial description of a procedure from the human scalp (Jewett \& Williston 1971), the meásurement of the brain-stem auditory evoked response (BAER) has become the most recent electrophysiological procedure to be integrated into testing protocols. This procedure subsequently had a major impact on the disciplines of audiology, otology and neurology, (Schwartz \& Berry, 1985). The development of the BAER was focused on two principal areas of application:

i. the evaluation and diagnosis of peripheral auditory problems and related pathology, and

ii. the assessment of the neural integrity of the acoustic nerve and caudal levels of the brain-stem afferent auditory pathway (Hecox \& Jacobson, 1984).

However, despite the reported robustness and stability of the $B A E R$ as a reliable assessment tool, it is critical to the effective use of this measurement to have diagnostic reference data that are collected within the individual laboratory or clinic. Several investigators have reported on the myriad of variables that can potentially alter one or more of the important parameters of the BAER, and hence lead to misinterpretation. It is, therefore, appropriately suggested by Schwartz \& Berry (1985), that it is not advisable for any clinician to depend on published diagnostic reference data for interpreting BAERs. This emerges from the opinion that there is a lack of uniformity in BAER measurement variables among investigators in various clinics and laboratories around the world.

Furthermore, although there is available information on diagnostic reference data on the normal BAER (see table 1, adapted from Schwartz \& Berry, 1985), such information is lacking in South Africa for any age or population group. Due to the absence of standard to specify recording parameters and methods used to measure the BAER, (this is clearly seen in table 1 whereby the various clinics and or laboratories differ in their test protocols), it is imperative that any clinic, including the Audiology Clinic at the University of Durban-Westville establishes its own diagnostic reference data based on its own test equipment and protocol. BAER interpretation maybe confounded by the influence of various factors, viz. differences in:

a. electrical and electromagnetic field variation between clinical/laboratory sites

b. the use of different stimuli

c. recording and analysis parameters

d. electrode placement

e. transducer type and

f. transducer placement.

The above differences may lead to small but significant changes in peak latency, amplitude and morphology. In addition a number of investigators (Beagly \& Sheldrake, 1978; Jerger \& Hall, 1980; and Stockard et al. 1978) reported that there is a marked sex effect on the normal BAER. Generally, females show shorter latencies and larger amplitudes than 
males. The difference is greater for peak $\mathrm{V}$ than for the earlier peaks, and also produces shorter IPLs within females. The clinical implication of this differential effect is that it is advisable to establish separate reference data for males and females to avoid misinterpretations (Hall, 1984). Therefore a study designed to elicit and to examine the BAER in a group of normal male and female subjects under controlled conditions would provide relevant information, leading to the establishment of suitable diagnostic reference data for the interpretation of BAER in the Audiology Clinic at the University of Durban-Westville.

\section{METHOD}

\section{Aim}

The aim of the study was to examine certain response characteristics of the BAER obtained in a selected group of normal hearing Indian male and female subjects. This would allow for the generation of diagnostic reference data for the interpretation of BAER at the Audiology Clinic at the University of Durban-Westville. The response characteristics of the normal BAER to be examined were as follows:

a. absolute latencies of peaks I to VI

b. relative (interpeak) latency (IPL) of peaks I-III, III-V and peaks I-V.

c. peak to trough amplitudes of peaks I and V

d. amplitude ratio obtained by comparing the absolute amplitudes of peak I and $\mathrm{V}$.

\section{SUBJECTS}

A total of 60 randomly selected subjects ( $\bar{X}$ age 20.7 years) comprising of 30 males ( $\overline{\mathrm{X}}$ age 21.3 years) and 30 females $(\overline{\mathrm{X}}$ age 20.3 years) contributed relevant data $\mathrm{fc}^{*}$ the purpose of this study. All subjects had normal hearing thresholds of 0 $26 \mathrm{~dB}$ for $\mathrm{AC}$ and $\mathrm{BC}$ for the test frequencies $250 \mathrm{~Hz}$ to $8000 \mathrm{~Hz}$, SRTs which were within $\pm 5 \mathrm{~dB}$ of the PTAs, speech discrimination scores of $92 \%$ to $100 \%$ at $35 \mathrm{dBsl}$, normal type A tympanograms, static compliance measures ranging between 0.28 and $2.5 \mathrm{cc}$, and contralateral acoustic reflex thresholds of between $70 \mathrm{dBsl}$ and $90 \mathrm{dBsl}$. Subjects also had a negative history of neurological abnormalities, consuming known ototoxic drugs and of excessive noise exposure as evidenced in the pre-test case history questionnaire (Govender, 1989). Furthermore, all subjects were right-handed as determined by a standardised handedness questionnaire (Lazarus, 1989), so as to select the right ear as the test ear. This was to ensure consistency in testing the same ear for all subjects.

\section{PROCEDURE}

All subjects were assessed in the supine position on a standard patient couch with appropriate head propping by using a pillow to minimize postural muscle activity around the head and neck regions (Chiappa et al. 1979). Subjects were encouraged to relax and to fall asleep during the recording session as this would reduce myogenic activity from influencing the responses. Therefore actual testing commenced only if subjects appeared to be relaxed or asleep.

The electrode sites were cleaned of all debris with omni-prep skin preparing paste and were slightly abraded to assist in reducing skin resistance. Self-adhesive silver-silver chloride electrodes were then arranged so that the electrical potential difference was measured between a pair of electrodes as suggested by Schwartz \& Berry (1985). The positive electrode was placed on the high forehead just below the hairline, the negative electrode was placed on the ipsilateral mastoid, while the contralateral mastoid was used as the site for the ground electrode. Prior to fixing the electrodes to the skin, standard EEG paste (colloidon) was applied between the surface of the electrode and the skin. The electrodes were then fixed firmly to the skin.

The recording electrodes were connected to a high gain $\left(10^{4}\right)$ differential, low noise, biological preamplifier (the Cadwell Quantum 84 preamplifier). The inter-electrode impedance was always below $3000 \Omega$ before testing commenced. Electrode activity was differentially filtered with a filter bandpass setting of $100 \mathrm{~Hz}$ to $3000 \mathrm{~Hz}$, as suggested by the Cadwell programme for BAER testing.

Alternating click stimuli with a duration of $100 \mu \mathrm{sec}$ were presented at a rate of 11.29 per sec at $70 \mathrm{dBHL}$ to the target right ear via a TDH-39 earphone, housed in an Amplivox freefield audiocup. The headphone position was held constant for all subjects. The no-test left ear was masked with $60 \mathrm{dBHL}$ of broadband noise so as to prevent its participation in obtaining the monaural BAER in the right ear.

The sweep time was set at 1 so that each division on the monitor screen represented $1 \mathrm{msec}$. Therefore, the BAFR was observed over a time frame of $10 \mathrm{msec}$ post-stimulus. A total of 2048 clicks was presented to ensure waveform build-up and clarity. The artifact rejection facility of the evoked potential system was switched on continuously so as to allow for automatic rejection of artifacts. All responses were recorded using the built in Alps printer of the evoked potential system.

All BAERs were elicited using the Cadwell Quantum 84 computer based, software run, auditory evoked response audiometer. Testing was conducted in a electro-magnetically screened anechoic sound treated chamber meeting noise level requirements set by ANSI (1977).

The actual test run was initiated as soon as all test parameters were set. Prior to any stimulus presentation, a control run was done to allow for comparing and identifying of true responses. At least two trials were done to ensure waveform repeatability and consistency. NB. See appendix 1 for a summary of the BAER test protocol used in this study.

\section{MEASUREMENTS MADE}

\section{Absolute latencies.}

The absolute latencies of peaks I to VI in msec were made from stimulus onset to the positive peak of each component wave of the BAER.

\section{Relative or Interpeak IPL latencies.}

The relative latencies in msec. of peaks I-III, III-V and I-V were recorded from the target ( $R$ ) ear. These were automatically calculated by the computer of the evoked response system.

\section{Absolute amplitudes.}

Peak to trough amplitudes of peaks I and $V$ in microvolts were measured. These were measured from the positive peak to the following negative trough of each of the named component waves of the BAER. 


\section{Amplitude ratio}

This ratio was obtained by comparing the amplitude of peak $V$ to that of peak I. The ratio was obtained by dividing the amplitude of peak $I$ by that of peak $V$, as suggested by Musiek \& Gollegly, (1985).

\section{ANALYSIS OF DATA}

The data were analysed by using conventional statistical procedures which allowed for the generation of means, ranges and standard deviations.

\section{RESULTS}

Table 2 summarises the statistical analyses made on the various latency and amplitude measurements obtained from the combined group $(\mathrm{N}=60)$.

Table 3 reflects a summary of the statistical analysis conducted for the various latency and amplitude measurements obtained among 30 female and 30 male subjects. This table was drawn up to specifically reflect the data for males and females separately.

Tables 2 and 3 therefore represent the diagnostic reference data related to the aim of the study

\section{DISCUSSION}

Table 2 provides a summary of the latency and amplitude measures made on the monaural BAER elicited in 60 normal hearing young students. The diagnostic reference data reflected in table 2 is to be discussed in relation to previously published data under the following headings:

\section{a) Absolute latencies}

Table 2 reflects the overall group absolute latency values for peaks I to VI. The obtained range extends from 2.08 to 7.49 msec in response to clicks presented at $70 \mathrm{dBnHL}$. The latency range for peaks I to $V$ was 2.8 to $6.00 \mathrm{msec}$. Beagley \& Sheldrake (1978), in reporting their "normative data" obtained from 5 male and 5 female subjects (age range 21-30 years) for peaks I to $V$ show a similar latency range of 2.1 to $6.1 \mathrm{msec}$ in response to clicks presented at $70 \mathrm{dBsl}$.

However, in comparing the findings of the above studies to those obtained in other clinics and laboratories (see table 1), it is evident that there are small variations between and among the values reported. These variations may be attributed to differences in intensity, polarity of clicks, repetition rates, filter setting and other aspects of test protocols used. The fact that these discrepancies between and among clinics and laboratories exist. highlights the need for each facility to generate its own diagnostic reference data.

An examination of the individual studies reported in table 1 reveals two major differences:

Table 1: Normative ABR latency data across 11 laboratories. Adapted from Schwartz \& Berry, (1985).

\begin{tabular}{|c|c|c|c|c|c|c|c|c|c|c|c|c|}
\hline \multirow{2}{*}{ Laboratory/Clinic } & \multirow{2}{*}{1} & \multirow{2}{*}{2} & \multirow{2}{*}{3} & \multirow{2}{*}{$\begin{array}{c}\text { Filter } \\
\text { settings } \\
(\mathrm{Hz})\end{array}$} & \multicolumn{8}{|c|}{ Wave latency (ms) } \\
\hline & & & & & $\mathrm{I}$ & II & III & IV & V & I-III & III-V & $\mathrm{I}-\mathrm{V}$ \\
\hline $\begin{array}{l}\text { Jewett and Williston, } \\
(1970)\end{array}$ & $60-75$ & $?$ & $?$ & $10-10,000$ & 1.5 & 2.6 & 3.5 & 4.3 & 5.1 & - & - & - \\
\hline $\begin{array}{l}\text { Lév and Sohmer, } \\
\qquad(1972)\end{array}$ & 65 & $?$ & $?$ & $250-5000$ & 1.5 & 2.5 & 3.5 & - & 5.0 & $\cdot$ & - & $\cdot$ \\
\hline $\begin{array}{l}\text { Picton et al. } \\
\quad(1974)\end{array}$ & 60 & $?$ & 10 & $10-3000$ & 1.5 & 2.6 & 3.8 & 5.0 & 5.8 & - & - & - \\
\hline $\begin{array}{l}\text { Starr and Achor, } \\
\text { (1975) }\end{array}$ & 65 & alt. & 10 & $100-3000$ & 1.6 & 2.8 & 3.8 & 4.8 & 5.5 & - & - & - \\
\hline $\begin{array}{l}\text { Stockard and Rossiter, } \\
\text { (1977) }\end{array}$ & 60 & rar. & 10 & $100-3000$ & 1.9 & 3.0 & 4,1 & 5.2 & 5.9 & 2.1 & 1.9 & 4.0 \\
\hline $\begin{array}{l}\text { Rosenhamer et al. } \\
\text { (1978) }\end{array}$ & 60 & $?$ & 16.6 & $180-4500$ & 1.7 & 2.9 & 3.9 & 5.2 & 5.9 & 2.26 & 2.0 & 4.27 \\
\hline $\begin{array}{l}\text { Row, } \\
\text { (1978) }\end{array}$ & 60 & $?$ & 10 & $100-3000$ & 1.9 & 2.9 & 3.8 & 5.1 & 5.8 & 1.97 & 1.97 & 3.94 \\
\hline $\begin{array}{l}\text { Gilroy and Lynn, } \\
\text { (1978) }\end{array}$ & 75 & $?$ & 11 & $150-3000$ & 1.55 & 2.67 & 3.60 & 4.69 & 5.40 & 2.05 & 1.9 & 3.83 \\
\hline $\begin{array}{l}\text { Beagley and Sheldrake, } \\
\text { (1978) }\end{array}$ & 70 & $?$ & 10 & $250-3200$ & 2.1 & 3.3 & 4.3 & 5.3 & 6.1 & 2.2 & 1.8 & 4.0 \\
\hline $\begin{array}{l}\text { Chiappa et al. } \\
(1979)\end{array}$ & 60 & alt. & 10 & $100-3000$ & 1.7 & 2.8 & 3.9 & 5.1 & 5.7 & 2.1 & 1.9 & 4.0 \\
\hline $\begin{array}{l}\text { Schwartz and Berry, } \\
\text { (1985) }\end{array}$ & $\begin{array}{c}75 \mathrm{~dB} \\
(\mathrm{nHL})\end{array}$ & rar. & 11.1 & $75-1500$ & 1.65 & 2.85 & 3.8 & 4.99 & 5.66 & 2.05 & 1.85 & 4.00 \\
\hline
\end{tabular}

$1=$ Stimulus intensity level in $\mathrm{dB}$

$2=$ Stimulus polarity

$3=$ Repetition rate $(\mathrm{cps})$ 
i. Some researchers did not always clearly define the reference intensity level for the clicks used, ie. whether the clicks were presented in $\mathrm{dBsl}, \mathrm{dBnHL}, \mathrm{dBspl}$ or $\mathrm{dBHL}$. This aspect is considered important as it reflects on exactly how loud a click is presented. Furthermore, it is well documented that there is a direct relationship between intensity level and the latency of the peaks obtained in the BAER (Rowe 1978, Stockard et al. 1978), ie. as the intensity of the click increases, the latencies of the peaks decreases and vice-versa (Moore 198:3). It is, therefore, suggested that investigators clearly define the reference intensity levels used. This suggestion is supported by the American Electroencephalic Society guidelines for clinical evoked potential studies (1984). Adherence to these guidelines would permit the uniform use of reference intensity levels and would allow for the comparison of reference data between and among clinics and laboratories.

ii. Investigators did not always clearly define the polarity of clicks used. BAERs are affected by the acoustic phase of clicks (Gastone et al. 1987). Rarefaction clicks evoke shorter peak I and V latencies (Coats \& Martin 1977); although these vary considerably among subjects. Condensation clicks, however, appear to delay peak I and V latencies (Schwartz \& Berry 1985), while alternating clicks do not seriously compromise BAERs, but serve to enhance the clarity of the response (Schwartz \& Berry, 1985). It is therefore recommended that investigators clearly define the acoustic phase of clicks used in the generation of "normal" reference data. This would facilitate the development of uniform procedures and hence allow for inter-facility comparison.

It is conceivable that among the variables mentioned earlier, both variation in intensity reference levels and the polarity of clicks used, may principally account for the differences in normal values reported in table $l$ and in this investigation.

In considering the appearance of individual peaks, all six peaks except for peak IV were consistently elicited in this investigation. Six subjects ( 2 males and 4 females) did not produce clear and measurable peak IV latencies. According to Beagley \& Sheldrake (1978), peak IV tends to be a more labile peak while Rowe (1978), states that it may, in some normal subjects be absent. This may he attributed to the fact that peak IV sometimes tends to fuse with peak $V$ thereby making it indistinct (Chiappa et al. 1979).

Peak I was consistently elicited in a latency range of 1.87 to 2.29 msec. This finding is consistent with Picton's (1986), recommended range of 1.4 to $2.5 \mathrm{msec}$. The mean of $2.08 \mathrm{msec}$, however, appears to be slightly delayed when compared to the means reflected in table 1 . Among the other variables mentioned earlier, this may be attributed to variations in testing and measurement protocols used in the different laboratories and clinics, particularly to differences in intensity levels of click phases used.

Generally, the absolute latencies of peaks II, III, IV and V show close approximation with those presented by Beagley \& Sheldrake (1978). However, in comparison with the other studies reflected in table 1 , these latencies appear to be slightly delayed, but the inter-facility standard deviations show close agreement. This provides support for the claim that the BAER is a stable and reliable measure at moderate to high intensity levels.
The inspection of the peak $\mathrm{V}$ latency, which, according to Schwart\% \&Berry (1985) and Stockard et al. (1978), should occur within $4.00 \mathrm{msec}$ after peak $\mathrm{I}$, reveals that the finding of this study is consistent with the above i.e. peak V (see table 1) had a mean latency period of $3.90 \mathrm{msec}$ after peak I. According to several researchers, e.g. Beagley and Sheldrake (1978), Stockard et al. (1978) and Picton (1986), peak V is the most consistent and prominent of the BAFRs, and is probably most useful diagnostically. Its appearance at a mean latency period of 6.00 msec in this study conforms well with findings reported by Stockard \& Rossiter (1977), at $5.9 \mathrm{msec}$, Rosenhamer et al. (1978), at $5.9 \mathrm{msec}$ and of Rowe, (1978) at 5.8 msec. Furthermore, the standard deviation of the peak $V$ latency as reported by all these researchers did not differ significantly from that of this study, i.e. a standard deviation of 0.23 . Clearly, peak V latency appears to be robust in character. It is reliable and stable even under varying measurement conditions. This contention is supported by the evidence presented in table 1 . It is, therefore, not surprising to note that peak $V$ (a rostral component of the BAER) has received widespread clinical attention in differential diagnosis of otoneurologic disorders, as well as for the estimation of hearing sensitivity (Schwartz \& Berry, 1985). The finding that there were some overall variations between this study and of those summarised in table 1, illustrates and highlights again the need for each clinic or test facility to establish its own diagnostic reference data.

\section{b) Relative or inter-peak latencies}

Table 2 also reflects the mean relative latency values obtained in the group of 60 normal hearing students. These include the values for the relative latencies of peaks I-III; III-V and peaks I$V$. Crucial to the differential diagnosis of space occupying lesions, either intrinsic or extrinsic to the brain-stem, is the time difference between peaks. These time differences are reflected by the time intervals between the following:

i. peaks I-III as representing peripheral transmission time from stimulus onset to the ponto-medullary junction in the lower pons (Stockard et al. 1978)

ii. peaks III-V as reflecting central transmission time from caudal pons to the midbrain (Schwartz \& Berry, 1985) and

iii. peaks $I-V$ as representing both peripheral and central transmission time from stimulus onset to the midbrain (Schwartz \& Berry. 1985).

Peripheral transmission time is determined by middle-ear function, cochlea mechanics, cochlea transduction, synaptic and cochlea nerve conduction velocity while central transmission time is associated with fibre conduction velocity and synaptic transmission of brain-stem tracts and nuclei (Cornacchia et al. 1983).

The mean relative latency values obtained in this investigation were as follows:

$$
\begin{aligned}
& \text { peaks I-III }=2.01 \mathrm{msec} \\
& \text { peaks III-V }=1.88 \mathrm{msec} \\
& \text { peaks } \mathrm{I}-\mathrm{V}=3.90 \mathrm{msec}
\end{aligned}
$$

These values coincide well with previously published data as reflected in table 1. Furthermore, these values fit in well with the suggested values presented by. Schwartz \& Berry (1985); these being $\pm 2 \mathrm{msec}$ for peaks I-III, and III-V and $\pm 4 \mathrm{msec}$. for peaks $\mathrm{I}-\mathrm{V}$ in normal hearing subjects. According to Rowe 
(1978), these relative latency values should not vary between and among laboratories and clinics using the same rate of stimulus presentation.

In summary, this investigation established relative latency values which are similar to those found in other clinics and laboratories having used a click stimulation rate of 10-12 clicks per sec (see table 1). The lack of variability in these measures between and among facilities using the same click rate, makes them robust measures of peripheral and central transmission time within the auditory system. 'Therefore, the interpeak latency measures are suitable for assessing pathologies which may affect the transmission of auditory impulses in the peripheral and brain-stem part of the auditory system.

\section{c) Absolute amplitudes}

In referring to table 2 , two absolute amplitude measures were considered in this investigation. These were the absolute amplitudes of peaks $I$ and $V$. The obtained values in $\mu V$ were used for the computation of the more clinically acceptable amplitude measure, i.e. the amplitude ratio of peak $V$ compared to peak I.

Table 2: Summary statistics for absolute and relative latencies in milli-seconds and absolute and relative amplitude measurements in micro-volts of the monaural BAER for the combined group. $(\mathrm{N}=\mathbf{6 0})$

\begin{tabular}{|c|c|c|c|}
\hline BAER Measures & \multicolumn{3}{|c|}{ Statistical Measures } \\
\hline Absolute Latencies (msec) & $\overline{\mathrm{X}}$ & Range & $\mathrm{SD}$ \\
\hline Peak I & 2.08 & $1.87-2.29$ & 0.08 \\
\hline Peak II & 3.02 & $2.17-3.37$ & 0,18 \\
\hline Peak III & 4.12 & $3.71-4.52$ & 0.17 \\
\hline Peak IV $(\mathrm{N}=54)$ & 5.13 & $4.42-5.83$ & 0.30 \\
\hline Peak V & 6.00 & $5.50-6.58$ & 0.23 \\
\hline Peak VI & 7.49 & $6.54-8.21$ & 0.32 \\
\hline \multicolumn{4}{|l|}{ Relative Latencies (msec) } \\
\hline Peak I-III & 2.01 & $1.69-2.41$ & 0.15 \\
\hline Peak III-V & 1.88 & $1.48-2.41$ & 0.18 \\
\hline Peak I-V & 3.90 & $3.45-4.37$ & 0.22 \\
\hline \multicolumn{4}{|l|}{ Absolute Amplitudes $(\mu \mathrm{V})$} \\
\hline Peak I & \multirow{3}{*}{$\begin{array}{l}0.17 \\
0.24\end{array}$} & \multirow{3}{*}{$\begin{array}{l}0.08-0.36 \\
0.08-0.50\end{array}$} & \multirow{3}{*}{$\begin{array}{l}0.05 \\
0.08\end{array}$} \\
\hline Peak V & & & \\
\hline Relative Amplitude $(\mu \mathrm{V})$ & & & \\
\hline Peak V:I & 1.50 & $0.55-3.85$ & 0.67 \\
\hline
\end{tabular}

N.B. For Peak IV, Combined group $\mathrm{N}=54$

Table 2 above reflects the diagnostic reference data for the various latency and amplitude measurements obtained from the combined group $(\mathrm{N}=60)$.

There is consensus among researchers, viz. Schwart\% \& Berry, (1985). Rowe, (1978), and Chiappa et al.(1979), that absolute amplitude measures are not normally distributed; are highly susceptible to myogenic activity and noise levels; are difficult to replicate, and are easily influenced by minor alterations in recording techniques. Consequently, the measurement of absolute amplitudes do not enjoy the stability and reliability of their latency counterparts (Schwartz \& Berry, 1985).
In this study, the mean peak I amplitude value was $0.17 \mu \mathrm{V}$ and that of peak $V$ was $0.24 \mu \mathrm{V}$. Chiappa et al. (1979), presented a mean peak amplitude value of $0,28 \mu \mathrm{V}$ and a mean peak $V$ value of $0.47 \mu \mathrm{V}$. Stockard et al. (1978), published a mean value of $0,23 \mu \mathrm{V}$ for peak I and $0,35 \mu \mathrm{V}$ for peak $\mathrm{V}$. It is not clear that there are no close approximations between and among reported measures.

These reported variations in amplitude measures between and among normal hearers may be attributed to the present system of signal averaging and use of artifact rejection (Fernandes, 1989). Theoretically, a wanted evoked potential is extracted from ongoing EEG by signal averaging and the use of artifact rejection. 'That is, by increasing the signal-to-noise ratio. Waveform and amplitude build up is, therefore, a product of time-locked averaging together with the rejection of other contaminating artifacts, e.g. myogenic and other cerebral activity.

It has been found that consensus among researchers on how much of averaging and/or artifact rejection is required before a response is judged as acceptable or not, is lacking. According to Hyde (1985), the choice of the number of clicks presented for averaging is often "based on popular consensus rather than on quantitative rationale". Due consideration has not been given to the influence of differences in "internal noise levels" among normal hearers when reference data are established. That is, some normal subjects may have higher internal noise levels, requiring longer periods of averaging with greater number of averages within a trial before eliciting an appropriate response than subjects who have lower internal noise levels (Hyde, 1985). Therefore a choice of either 1048,200 or 2048 clicks to elicit a suitable averaged response may not be appropriate for all normal hearers. Furthermore, since the amplitude of a response is partly dependent on the number of averages that occur in a trial, it is reasonable to assume that response amplitudes will differ between and among individuals. This therefore may account for the variability in amplitude measurements that are reported in the literature.

Similarly, the use and control of artifact rejection to eliminate unwanted noise is not consistent in studies that have reported on normal amplitude values. It is therefore not surprising to find variations in the reported amplitude values between and among studies.

The consistent and approriate application of signal averaging and management of artifact rejection needs to be given careful attention in future research. Attention needs to be focused on decisions pertaining to the:

i. Actual number of averages required in a trail (i.e. 1048 , 2000 or 2048 clicks) before a response is regarded as representative of a "true neurogenic" response.

ii. Use and control of artifact rejection so that the final response is truely representative of the BAER without being contaminated by other artifacts.

A reasonable course of action, is to set the artifact rejection limits so that little of the "well behaved" (low variance) activity is rejected, while all of the high variance (bursts of electromyogenic noise) activity is. This may be done by "tuning" the rejection level while observing the displayed activity, so that only about $5-10 \%$ of the good activity is rejected. Perhaps, the manufacturers of evoked potential systems need to incor- 
porate additional desirable features that will allow for the display of the input EEG during averaging, rejection of trails in which large voltage artifacts occur and an assessment of amplitude variability within an averaging run.

The above may assist in establishing appropriate reference data for amplitudes which may be used routinely in BAER interpretation. Thereafter, such reference data should be applied widely to assess how otoneurologic pathologies influence the measures, and to document the obtained patterns for ongoing comparisons.

\section{d) Relative amplitude - the peak V:I amplitude ratio}

Table 2 reveals, that the mean amplitude ratio obtained in this investigation was 1.50 . This is consistent with the findings of Chiappa et al. (1979); Rowe (1978), and that of Starr \& Achor (1975), who have all reported that a value greater than 1.00 be considered as normal. In order to detect abnormality, Musiek et al. (1984), state that amplitude ratio should be less than 1.00. Stockard et al. (1978), however, state that a complete absence of peak $V$ in the presence of peak $I$ is an indication of relative amplitude abnormality.

Differing in this opinion, Starr \& Achor (1975), state that a peak V:I a mplitude ratio of less than 0,5 at $55 \mathrm{dBs}$ is abnormal. Later in 1978, Stockard et al. suggested that the peak V absolute amplitude value which is reduced by more than $3 \mathrm{sd}$ from the normal mean, together with a peak I amplitude that is larger than peak $V$, and an inter-trial variation of less than $10 \%$ are all necessary for the peak V:I amplitude ratio to be defined as abnormal. Chiappa et al. (1979), agree with Starr \& Achor (1975), in the 10 of their 104 normal subjects displayed a peak I amplitude which was larger than peak $V$. The findings of this investigation are in part agreement with Starr \& Achor (1975), and with Chiappa et al. (1979), since 12 subjects (5 females and 7 males) displayed peak I amplitudes which were larger than peak V, although the overall mean was 1.50 . The observed differences in amplitude ratios appear to be due to normal variations that occur within and among normal individuals. This contention is in keeping with Stockard et al.'s (1977), statement that "alterations of BAFR morphology in the absence of quantifiable latency or absolute amplitude abnormality are not considered abnormal per se, because of the variability of BAER waveforms within and among normal individuals."

However, Schwartz \& Berry (1985), are of the opinion that there is a dearth of well documented literature concerning the use of the V:I amplitude ratio in a large pathologic population.
They suggest that considerable research is needed on the confounding effects of such variables as stimulus polarity, repetition rates, filter characteristics, electrode sites etc., prior to the general use of this measure in clinical practice. The investigator concurs with the above recommendation. Due consideration should also be given to inter and intra individual variations when examining amplitude data. Furthermore, and improvement in signal averaging and artifact control may aid in resolving the issue of obtaining variable amplitude measures in normal hearers.

\section{SEPARATE DIAGNOSTIC REFERENCE DATA FOR MALES AND FEMALES}

In response to the suggestion made by several researchers, viz. Stockard et al. (1978), (1979); Jerger \& Hall (1980), and Jerger \& Johnson (1988), that diagnostic reference data be established separately for males and females, the raw data was further treated to reflect this separation.

Table 3, reflects the means, ranges and standard deviations for the various BAER measurements as obtained from 30 females and 30 males.

On inspection and comparison of the mean absolute latency values obtained for the two groups, it is evident that for all six peaks, females tended to show shorter latency values than males. This is also evident for the peak I-III and peak I-V relative latency values.

The absolute and relative amplitude measures show no such differences, implying that there are no observable differences between sexes for these measures in this investigation. However, further research focusing on the appropriate use of signal averaging and artifact rejection may produce realistic amplitude measures in normal hearers. Once this has been achieved, it is suggested that the effect of sex difference on amplitude measurements be reassessed.

The question of whether there is a statistically significant sex difference effect on the normal BAER, needs to be researched further.

In the interim, the fact that there are observed latency differences between the sexes as seen in table 3 , is supportive of the suggestion that separate diagnostic reference data be established for the two sexes. The establishment of such data, would prevent the clinician from applying inappropriate sex related reference data to interpret the BAER. 
Table 3: Summary statistics for absolute and relative latencies in milli-seconds and absolute and relative amplitude measurements in micro-volts of the monaural evoked BAER in females $(N=30)$ and males $(N=30)$

\begin{tabular}{|c|c|c|c|c|c|c|}
\hline \multirow{3}{*}{$\begin{array}{l}\text { BAER Measures ( } \mathrm{P}=\text { Peak }) \\
\text { Absolute Latencies }\end{array}$} & \multicolumn{6}{|c|}{ Statistical Measures } \\
\hline & \multicolumn{3}{|c|}{ Females $(\mathrm{N}=30)$} & \multicolumn{3}{|c|}{ Males $(\mathrm{N}=30)$} \\
\hline & $\overline{\mathrm{X}}$ & Range & SD & $\overline{\mathrm{X}}$ & Range & $\mathrm{SD}$ \\
\hline P I & 2.06 & $1.87-2.19$ & 0.07 & 2.11 & $1.92-2.29$ & 0.10 \\
\hline P II & 3.02 & $2.71-3.29$ & 0.14 & 3.4 & $2.79-3.37$ & 0.14 \\
\hline P III & 4.08 & $3.71-4.52$ & 0.18 & 4.16 & $3.87-4.50$ & 0.16 \\
\hline P IV & 5.10 & $4.42-5.83$ & 0.37 & 5.17 & $4.58-5.52$ & 0.21 \\
\hline P V & 5.98 & $5.54-6.58$ & 0.24 & 6.03 & $\cdot 5.50-6.42$ & 0.22 \\
\hline P VI & 7.44 & $6.75-7.96$ & 0.30 & 7.55 & $6.54-8.21$ & 0.33 \\
\hline \multicolumn{7}{|l|}{ Relative Latencies } \\
\hline P I-III & 2.00 & $1.74-2.41$ & 0.16 & 2.03 & $1.69-2.37$ & 0.15 \\
\hline P III-V & 1.87 & $1.48-2.41$ & 0.18 & 1.87 & $1.56-2.29$ & 0.17 \\
\hline $\mathrm{P} I-\mathrm{V}$ & 3.84 & $3.49-4.37$ & 0.22 & 3.91 & $3.45-4.33$ & 0.23 \\
\hline \multicolumn{7}{|l|}{ Absolute Amplitude } \\
\hline$P_{I}$ & 0.17 & $0.08-0.36$ & 0.05 & 0.16 & $0.09-0.29$ & 0.05 \\
\hline $\mathrm{PV}$ & 0.25 & $0.09-0.44$ & 0.08 & 0.22 & $0.08-0.50$ & 0.08 \\
\hline \multicolumn{7}{|l|}{ Relative Amplitude } \\
\hline P V:1 & 1.50 & $0.74-2.50$ & 0.51 & 1.50 & $0.55-3.85$ & 0.81 \\
\hline
\end{tabular}

N.B. For peak IV: Female no. $=26$

Male no. $=28$

Table 3 represents the means, ranges and standard deviations for the various BAER measurements as obtained for the females and males respectively.

\section{CONCLUSION}

Diagnostic reference data were established for both the combined group $(\mathrm{N}=60)$ and separately for females and males. Similarities an differences between this study and of those reported in the literature were noted and discussed. The similarities in absolute latency méasures were attributed to close approximations between testing protocols used, whilst variations were primarily related to, among other variables, différences in reference intensity levels and the polarity of clicks.

Despite the difference between this study and of those reflected in table 1 , the absolute latency of peak $V$ remained resistant to variations in stimulus, recording and "normal subject" variables. Therefore this measure appears to be robust and maybe reliably used in otoneurological diagnosis and for estimating hearing sensitivity.

The relative latency values generated are consistent with those reported in the literature (see table 1), and this is attributed to the fact that click presentation rates used are similar, i.e. 10-12 clicks per sec., in each of the studies. It is therefore suggested that clinicians may confidently use these measures to assess otoneurological pathologies that may upset the conduction of impulses in the auditory periphery (e.g. multiple sclerosis), provided that the click rate used is 10 to 12 per sec.

Differences in amplitude measures between this study and among other studies were noted. These variations were, among other factors, attributed to the manner in which signal averaging and artifact rejection have been manipulated in obtaining the average BAER. Further research in this respect has been suggested. However, the relative amplitude value of 1.50 obtained in this study is consistent with those reported in the literature. This implies that the RA measure is less variable in normals and therefore, may be used as a more sensitive measure of brain-stem auditory function than absolute amplitude measures.

In view of the demonstrated differences in reference data between and among clinics and laboratories, the writer is of the opinion that clinicians should exercise caution in using reference data established elsewhere, especially if reported testing protocols differ in stimulation, recording and normal subject variables, e.g. sex. The observation that there were differences between the sexes is strongly supportive of the suggestion that separate reference data be established for the sexes (Stockard et al. 1978; Jerger \& Hall, 1980). This would allow for the accurate clinical interpretation of the BAER obtained in the two sex groups. Therefore it is recommended that each clinic generates its own reference data commensurate with its needs.

Furthermore, noting that this study fell short of giving due consideration to age-related data across the continuum, interaural latency differences, use of different repetition rates, stimulus intensity reference levels and click polarity, future research considering the above, needs to be conducted to extend the present reference data base.

The need for consensus to be reached among researchers and clinicians with respect to test protocols used in BEAR testing cannot be overemphasized. Perhaps, an international conference involving the various disciplines that use this test pro- 
cedure should be held, in order to formulate a standard guideline or protocol for the use of interpretation of the BAER. This would facilitate inter-clinic and/or laboratory comparisons, and perhaps aid in resolving some of the controversies that exist in BAER testing and interpretation.

In the interim, it is important the researchers and clinicians clearly define the parameters of their test protocols in establishing reference data. In addition, such data should be applied within populations having known otoneurological pathologies to assess the extent to which the reference data is able to differentiate normal from pathological ears. The latter is also applicable to the reference data generated in this study.

\section{REFERENCES}

American Electroencepalographic Society. Guidelines for clinical evoked potential studies. Joumal of Clinical Neurophysiology, 1 , $3-53,1984$.

American National Standards Institute Standard criteria for permissibility of ambient noise during audiometric testing. ANSI 53:1977 New York: American National Standards Institute, 1977.

Beagley, H.A., and Sheldrake, J.B. Differences in brain-stem response latency with age and sex. British Journal of Audiology. 12, 69-77, 1978

Chiappa, K.H., Gladstone, K.J., and Young, R.R. Brain-stem auditory evoked responses. Studies of waveform variations in 50 normal human subjects. Archives of Neurology, 36, 81-87, 1979.

Coats, A.C. and Martin, J.L. Human auditory nerve action potentials and brain-stem evoked responses. Archives of Otolaryngology. $103,605-622,1977$.

Cornacchia, L., Martini, A., and Morra, B. Air and bone conduction brain-stem responses in adults and infants. Audiology, 22,430$437,1983$.

Fernandes, C.M.C. EN'I Department, Medical School, University of Natal, Personal Communication, 1989.

Gastone, G.C., and Grigg, M.M. Auditory evoked potentials. In E: Niedermeyer and F. Lopes da Silva (eds.), Electroencephalography: Basic principles, Clinical applications and Related folds. Baltimore: Urban and Schwarzenberg. 1987.
Govender, C.D. An investigation into the effects of sex difference and contralateral masking on the monaural auditory evoked response (BAER) obtained in a group of normal hearing Indian undergraduate university students. Unpublished masters dissertation, University of Durban-Westville, 1989.

Hall, J.W. Auditory brain-stem audiometry. In J. Jerger (ed.), Hearing Disorders in Adults. San Diego: College-Hill Press, Inc, 1984.

Hecox, K., and Jacobson, J.'T'. Auditory evoked potentials. In J.L. Northern (ed.), Hearing Disorders. Boston: Iittle-Brown, 1984.

Hyde. M.L. Instrumentation and Signal Processing. In J.'I'. Jacobson (ed.), The Auditory Brain-stem Response, San Diego: College-Hill Press, Inc, 1985.

Jerger, J., and Hall, J. Effects of age and sex on auditory brain-stem response. Archives of Otolaryngology 106, 387-391, 1980.

Jerger, J., and Johnson, K. Interactions of age, gender and sensorineural hearing loss on ABR latency. Ear and Hearing, 9. $190-197,1988$.

Jewett, D.L., and Williston, J.S. Auditory evoked responses for fields averaged from the scalp of humans. Brain, 94, 681-696, 1971.

Lazarus, T. Annets Handedness Questionnaire. Modified and adapted for S.A. use. Unpublished, available from author, Department of Psychology, University of Durban-Westville, 1989.

Moore, E.J. Effects of stimulus parameters. In E.J. Moore (ed.), Bases of unditory brain-stem evoked responses. New York: Grune and Stratton, 1983.

Musiek, F.E., and Gollegly, K.M. ABR in Eighth nerve and low brainstem lesions. In J.T. Jacobson(ed.), The auditory brain-stem response. San Diego: College-Hill Press, 1985.

Musiek, F.E., Kibbe, K., Rackliffe, L., and Weider, D.J. The auditory brain-stem response I-V amplitude ratio in normal, cochlear and retrocochlear ears. Ear and Hearing, 5, 52-55, 1984.

Picton, T.W. Abnormal brain-stem auditory evoked potentials: A tentative classification. In R.Q. Cracco and I. Bodis-Wollner (Eds.), Frontiers of dinical neuroscience. Evoked Potentials, Vol. 3. New York: Alan R. I,iss Inc, 1986.

Rosenhamer, H., Lindstrom, B., and Lundborg, J. On the use of click evoked electric brain-stem responses in audiological diagnosis. i. The variability of the normal response. Scandinavian Audiology. $7,197-206,1978$.

Rowe, M.J. Normal variability of the brain-stem auditory evoked response in young and old subjects. Electroencephalography and Clinical Neurophysiology, 44, 459-470, 1978.

Schwartz, D.M., and Berry, G.A. Normative aspects of the ABR. In J.T. Jacobson (ed.), 'The Auditory Brain-stem Response. San Diego: College-Hill Press, 1985.

Starr,A., and Achor, J. Auditory brain-stem responses in neurological disease. Archives of Neurology, 32, 761-768, 1975.

Stockard, J.J., and Rossiter, V.S. Clinical and pathologic correlates of brain-stem auditory response abnormalities. Neurology, 27, $316-325,1977$.

Stockard, J.J., Stockard, J.E., and Sharbrough, F.W. Non-pathologic| factors influencing brain-stem auditory evoked potentials.! American Journal of Electroencephalographic Technology. 18, 171-209,1978. 
APPENDIX I

BEAR - TEST PROTOCOL

TECHNICAL AND PROCEDURAL CONSIDERATIONS

STIMULUS

TRANSDUCER

ELECTRODES

EVOKED RESPONSE AUDIOMETER

ELECTRODE SITES

clicks $-100 \mu$ sec. duration

electrodynamic TDH-39P earphones housed in free field audio-cups.

: $\quad$ self-adhesive silver-silver chloride.

: Cadwell Quantum 84

: positive $-\mathrm{Fz}_{z}$ high forehead

negative - ipsilateral mastoid

ground - contralateral mastoid

POLARITY

: alternating

REPETITION RATE

: $\quad 11,29$ per sec

FILTER PASS BAND

$100 \mathrm{~Hz}-3000 \mathrm{~Hz}$

SWEEP TIME

: $\quad 1$ division $=1 \mathrm{msec}$

TIME FRAME

: $\quad 10 \mathrm{msec}$ post-stimulus

NO. OF CLICKS PER TRIAL

2048

NO. OF TRIALS

CONTRALATERAL MASKING

LEVEL OF TEST EAR STIMULUS

Minimum-Two to ensure waveform repeatability.

$60 \mathrm{dBHL}$

ARTIFACT REJECTION

kept constant at $70 \mathrm{dBnHL}$

RECORDING OF RESPONSES

TÉST ENVIRONMENT

switched on

by built in ALPS Printer

ANECHOIC Chamber - electromagnetically screened low noise levels ANSI (1979).

PATIENT STATE

appeared to be relaxed or asleep lying in a supine position on a standard patient couch.

N.B.: A control run prior to stimulation was done to allow for comparing and identifying true responses. 


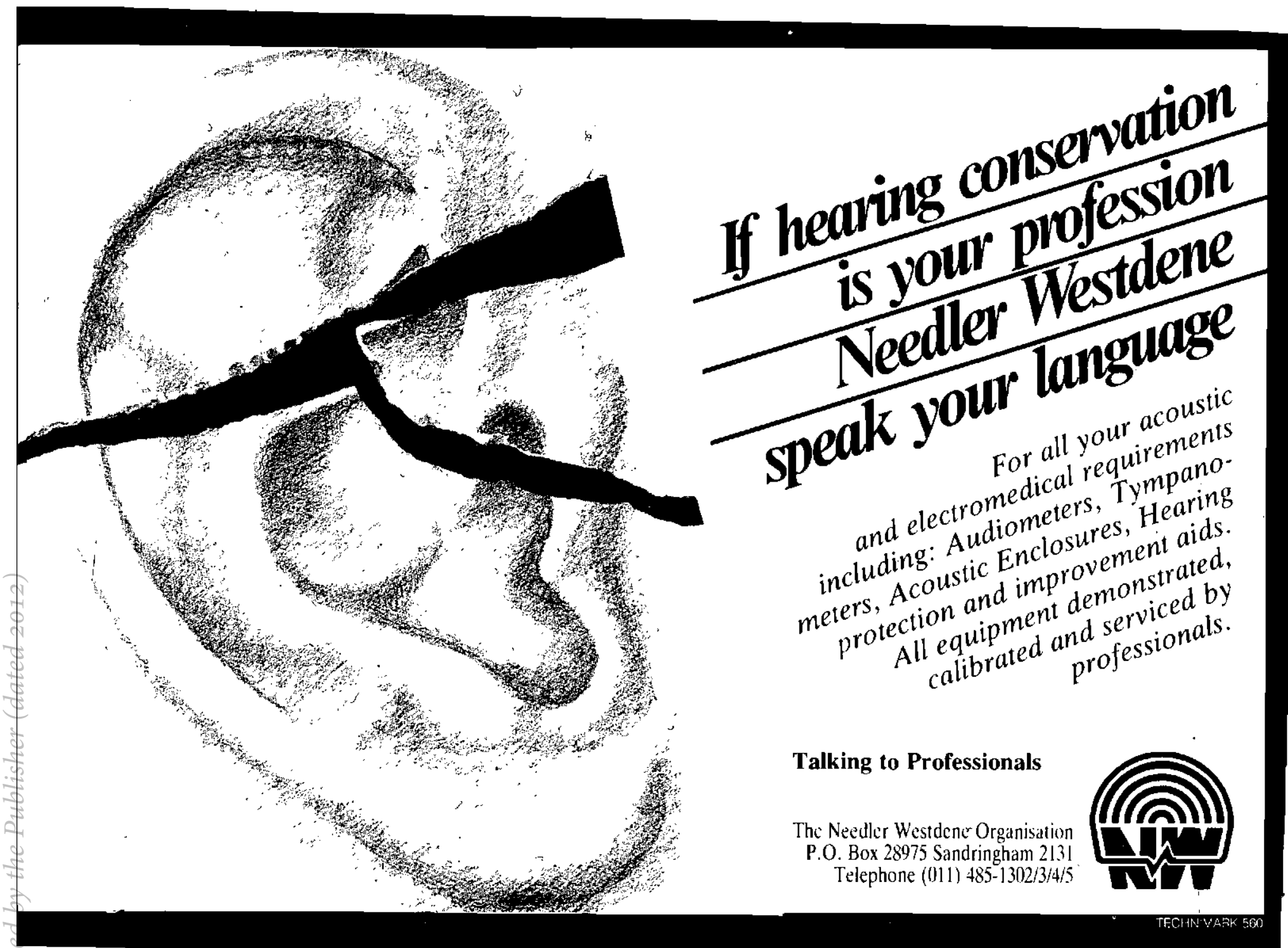

